\title{
Approximate solution of second-order fuzzy boundary value problem
}

\author{
Mine Aylin Bayrak \\ Kocaeli University, Department of Mathematics, Kocaeli,Turkey \\ Received: 21 February 2017, Accepted: 15 July 2017 \\ Published online: 17 August 2017.
}

\begin{abstract}
In this paper, a new approach is proposed based on the Adomian Decomposition Method(ADM) with Green's function in order to find a solution for the second-order fuzzy boundary value problem under generalized $H$-differentiability. The proposed technique divides the domain and builds on Green's function before installing the modified recursive scheme. Some examples are presented to illustrate the efficiency of the proposed technique.
\end{abstract}

Keywords: Fuzzy second-order boundary value problem, Adomian decomposition method, Green function's, Generalized differentiability.

\section{Introduction}

The fuzzy differential, integral equations and integro-differential equations are play very important roles in the fuzzy analysis theory. In many physical problems some quantities of the boundary values can be uncertain and they can be modeled by fuzzy numbers or fuzzy functions. The concept of fuzzy numbers was introduced by Zadeh [11,30]. By using $H$-derivative, several articles $[26,12,13]$ have demonstrated the solution to the fuzzy differential equations. However, Bede and Gal [7] introduced a generalized definition for fuzzy derivative and a fuzzy-number-valued function. Recently, some mathematicians have become interested in finding the solutions to the fuzzy linear and nonlinear differential equation, and fuzzy integral equation [19,18, 15,5,3,25,4,24,8]. The goal of this paper is to introduce an new approach by using generalized concepts $[2,21,23,22,20,14,16,9,1]$ for the solution of second-order fuzzy boundary value problem

$$
\widetilde{y}^{\prime \prime}=F\left(x, \tilde{y}(x), \tilde{y}^{\prime}(x)\right)
$$

with the boundary conditions

$$
\widetilde{y}^{\prime}\left(x_{0}\right)=A, \widetilde{y}^{\prime}\left(x_{2}\right)=B
$$

where $x_{0}, x_{2}$ has constant values, $A, B$ are fuzzy functions.

The structure of this paper is as follows: In Section 2, basic notations and definitions of fuzzy calculus are given. In Section 3, Eqs. (1) - (2) is solved using the Adomian decomposition method and Green's function under generalized differentiability. Convergence of the proposed technique is proven in Section 4. Finally, in Section 5. The efficiency of the technique is shown by solving some numerical examples and a brief conclusion is given in Section 6 .

\section{Preliminaries}

There are various definitions for the concept of fuzzy numbers $[6,10]$.

\footnotetext{
* Corresponding author e-mail: aylin@ kocaeli.edu.tr
} 
Definition 1. A fuzzy number is a function $u: R \rightarrow[0,1]$ which satisfies the following properties:

(i) $u$ is normal;

(ii) $u$ is convex fuzzy set;

(iii) $u$ is upper semi-continuous on $R$;

(iv) $[A]^{0}=\operatorname{supp}(A)=\{x \in R \mid A(x) \geq 0\}$ is compact, where $\bar{A}$ denotes the closure of $A$.

Definition 2. An arbitrary fuzzy number $\tilde{u}$ in the parametric form is represented by an ordered pair of functions $(\underline{u}, \bar{u})$ of functions $(\underline{u}(r), \bar{u}(r))$, which satisfy the following requirements:

(i) $\underline{u}(r)$ is a bounded non-decreasing left continuous function in $(0,1]$, and right continuous at 0 ;

$(i i) \bar{u}(r)$ is a bounded non-increasing left continuous function in $(0,1]$, and right continuous at 0 ;

(iii) $\underline{u}(r) \leq \bar{u}(r)$ for all $0 \leq r \leq 1$.

The set of all fuzzy numbers is denoted by $F$ and it is a convex cone.

Definition 3. For arbitrary $\widetilde{u}=(\underline{u}(r), \bar{u}(r))$ and $\widetilde{v}=(\underline{v}(r), \bar{v}(r)), \quad 0 \leq r \leq 1$, and scalar $k$, addition, subtraction, scalar product by $k$, and multiplication is defined as following

Addition:

$\underline{\underline{(u+v)}}(r)=\underline{u}(r)+\underline{v}(r)$,

$\overline{\overline{(u+v)}}(r)=\bar{u}(r)+\bar{v}(r)$,

Subtraction:

$\underline{(u-v)}(r)=\underline{u}(r)-\underline{v}(r)$,

$\overline{\overline{(u-v)}}(r)=\bar{u}(r)-\bar{v}(r)$,

Scalar Product:

$k \widetilde{u}= \begin{cases}(k \underline{u}(r), k \bar{u}(r)), & k \geq 0, \\ (k \bar{u}(r), k \underline{u}(r)), & k<0\end{cases}$

Multiplication:

$\underline{u v}(r)=\min \{\underline{u}(r) \underline{v}(r), \underline{u}(r) \bar{v}(r), \bar{u}(r) \underline{v}(r), \bar{u}(r) \bar{v}(r)\}$

$\overline{u v}(r)=\max \{\underline{u}(r) \underline{v}(r), \underline{u}(r) \bar{v}(r), \bar{u}(r) \underline{v}(r), \bar{u}(r) \bar{v}(r)\}$

Definition 4. For arbitrary fuzzy numbers $\widetilde{u}, \widetilde{v} \in F$, the distance (Hausdorff metric) is used [17]

$D(u(r), v(r))=\max \left\{\sup _{r \in[0,1]}|\underline{u}(r)-\underline{v}(r)|, \sup _{r \in[0,1]}|\bar{u}(r)-\bar{v}(r)|\right\}$,

and it is shown [27] that $(F, D)$ is a complete metric space and the following properties are known:

$D(\widetilde{u}+\widetilde{w}, \widetilde{v}+\widetilde{w})=D(\widetilde{u}, \widetilde{v}), \quad \forall \widetilde{u}, \widetilde{v} \in F$,

$D(k \tilde{u}, k \widetilde{v})=|k| D(\widetilde{u}, \widetilde{v}), \quad \forall k \in R, \widetilde{u}, \widetilde{v} \in F$,

$D(\widetilde{u}+\widetilde{v}, \widetilde{w}+\widetilde{e}) \leq D(\widetilde{u}, \widetilde{w})+D(\widetilde{v}, \widetilde{e}), \quad \forall \widetilde{u}, \widetilde{v}, \widetilde{w}, \widetilde{e} \in F$

Definition 5. If the fuzzy function $\widetilde{f}(t)$ is continuous in the metric D, then definite integral exists [17], and also, $\begin{aligned}\left(\underline{\left.\frac{\left(\int_{a}^{b} f(t, r) d t\right.}{\bar{b}}\right)}\right. & =\int_{a}^{b} \underline{f}(t, r) d t \\ \overline{\left(\int^{b} f(t, r) d t\right)} & =\int_{a}^{b} \bar{f}(t, r) d t\end{aligned}$

Definition 6. Let be $\widetilde{x}, \tilde{y} \in F$. If there exists $\widetilde{z} \in F$ such that $\widetilde{x}=\widetilde{y} \oplus \widetilde{z}$, then $\widetilde{z}$ is called the H-difference of $\widetilde{x}$ and $\widetilde{y}$ and is denoted by $\tilde{x} \ominus \tilde{y}[7]$.

Proposition 1. If $\widetilde{f}:(a, b) \rightarrow F$ is a continuous fuzzy valued function, then $\widetilde{g}(x)=\int_{a}^{x} \widetilde{f}(t) d t$ is differentiable using a derivative $\widetilde{g}^{\prime}(x)=\widetilde{f}(x)[7]$

Definition 7.[7] Let $\tilde{f}:(a, b) \rightarrow F$ and $x_{0} \in(a, b) . \widetilde{f}$ is a generalized differentiable in $x_{0}$ (Bede-Gal differentiability), if there exists an element $\widetilde{f}^{\prime}\left(x_{0}\right) \in F$, such that. 
(i) for all $h>0$ sufficiently small, $\exists \widetilde{f}\left(x_{0}+h\right) \ominus \widetilde{f}\left(x_{0}\right), \exists \widetilde{f}\left(x_{0}\right) \ominus \widetilde{f}\left(x_{0}-h\right)$ and the following limits hold. $\lim _{h \rightarrow 0} \frac{\tilde{f}\left(x_{0}+h\right) \ominus \widetilde{f}\left(x_{0}\right)}{h}=\lim _{h \rightarrow 0} \frac{\tilde{f}\left(x_{0}\right) \ominus \widetilde{f}\left(x_{0}-h\right)}{h}=\widetilde{f}^{\prime}\left(x_{0}\right)$ or

(ii) for all $h>0$ sufficiently small, $\exists \widetilde{f}\left(x_{0}\right) \ominus \widetilde{f}\left(x_{0}+h\right), \exists \widetilde{f}\left(x_{0}-h\right) \ominus \widetilde{f}\left(x_{0}\right)$ and the following limits hold. $\lim _{\substack{h \rightarrow 0 \\ \text { or }}} \frac{\widetilde{f}\left(x_{0}\right) \ominus \widetilde{f}\left(x_{0}+h\right)}{-h}=\lim _{h \rightarrow 0} \frac{\widetilde{f}\left(x_{0}-h\right) \ominus \widetilde{f}\left(x_{0}\right)}{-h}=\widetilde{f}^{\prime}\left(x_{0}\right)$

(iii) for all $h>0$ sufficiently small, $\exists \widetilde{f}\left(x_{0}+h\right) \ominus \widetilde{f}\left(x_{0}\right), \exists \widetilde{f}\left(x_{0}-h\right) \ominus \widetilde{f}\left(x_{0}\right)$ and the following limits hold. $\lim _{\substack{h \rightarrow 0 \\ o r}} \frac{\widetilde{f}\left(x_{0}+h\right) \ominus \widetilde{f}\left(x_{0}\right)}{h}=\lim _{h \rightarrow 0} \frac{\widetilde{f}\left(x_{0}-h\right) \ominus \widetilde{f}\left(x_{0}\right)}{-h}=\widetilde{f}^{\prime}\left(x_{0}\right)$

(iv) for all $h>0$ sufficiently small, $\exists \widetilde{f}\left(x_{0}\right) \ominus \widetilde{f}\left(x_{0}+h\right), \exists \widetilde{f}\left(x_{0}\right) \ominus \widetilde{f}\left(x_{0}-h\right)$ and the following limits hold:

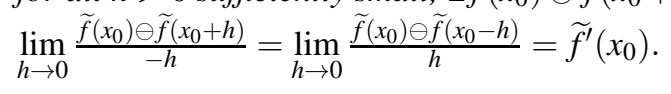

Definition 8. Let $\widetilde{f}:(a, b) \rightarrow F . \widetilde{f}$ is (i)-differentiable in $(a, b)$ if $\widetilde{f}$ is differentiable in the sense (i) of Definition (7) and similar in (ii), (iii) and (iv) differentiability.

Lemma 1.[7] For $x_{0} \in R$, the fuzzy differential equation $\widetilde{y}=\widetilde{f}(x, y), \quad \widetilde{y}\left(x_{0}\right)=\widetilde{y_{0}} \in F$, where $\widetilde{f}: R \times F \rightarrow F$ is supposed to be continuous, if is equivalent to one of the integral equations. $\widetilde{y}(x)=\widetilde{y}_{0}+\int_{x_{0}}^{x} \widetilde{f}(t, \widetilde{y}(t)) d t, \quad \forall x \in\left[x_{0}, x_{1}\right]$, or

$\widetilde{y}(x)=\widetilde{y}_{0}+(-1) \int_{x_{0}}^{x} \widetilde{f}(t, \widetilde{y}(t)) d t, \quad \forall x \in\left[x_{0}, x_{1}\right]$

On some interval $\left(x_{0}, x_{1}\right) \subset R$, under the differentiability condition, $(i)$ or (ii), respectively.

Remark. In the case of a strongly generalized $H$-differentiability, as to the fuzzy differential equation $\widetilde{y}=\widetilde{f}(x, y)$, then two different integral equations may be attached, while in the case of $H$-differentiability, only one may be attached.

The second integral equation in Lemma (1) can be written in the form [7]:

$\widetilde{y}(x)=\widetilde{y}_{0} \ominus(-1) \int_{x_{0}}^{x} \widetilde{f}(t, \widetilde{y}(t)) d t$

Theorem 1.[7] Suppose that the following conditions hold.

(a) Let $R_{0}=\left[x_{0}, x_{0}+p\right] \times \bar{B}\left(y_{0}, q\right), \quad p, q>0, \widetilde{y_{0}} \in F$, where $\bar{B}\left(y_{0}, q\right)=y \in F: D\left(y, y_{0}\right) \leq q$ denotes a closed ball in $F$ and let $\widetilde{f}: R_{0} \rightarrow F$ be a continuous function such that $D(\widetilde{0}, \widetilde{f}(x, y))=\|\widetilde{f}(x, y)\| \leq M$ is used for all $(x, y) \in R_{0}$.

(b) Let $\widetilde{g}:\left[x_{0}, x_{0}+p\right] \times[0, q] \rightarrow R$ such that $\widetilde{g}(x, 0) \equiv 0$ and $0 \leq \widetilde{g}(x, u) \leq M_{1}, \quad \forall x \in\left[x_{0}, x_{0}+p\right], \quad 0 \leq u \leq q$. Such that $g(x, u)$ is non-decreasing in $u$ and $g$ and such that the initial value problem $u^{\prime}=g\left(x, u(x), u\left(x_{0}\right)\right)=0$ has the only solution $u(x) \equiv 0$ on $\left[x_{0}, x_{0}+p\right]$.

(c) $D(f(x, y), f(x, z)) \leq g(x, D(y, z)), \quad \forall(x, y),(x, z) \in R_{0}$ and $D(y, z) \leq q$.

(d) There exists $d>0$ such that for $x \in\left[x_{0}, x_{0}+d\right]$ the sequence $\bar{y}_{n}:\left[x_{0}, x_{0}+d\right] \rightarrow F$ given by $\widetilde{\bar{y}}_{0}(x)=\widetilde{y}_{0}, \quad \widetilde{y}_{n+1}(x)=$ $\widetilde{y_{0}} \ominus(-1) \cdot \int_{x_{0}}^{x} \widetilde{f}\left(t, \widetilde{\bar{y}}_{n}(t)\right) d t$ is defined for any $n \in N$. Then the fuzzy initial value problem $\widetilde{y}=\widetilde{f}(x, y), \quad \widetilde{y}\left(x_{0}\right)=\widetilde{y}_{0}$, has two solutions (one (i)-differentiable and the other one (ii)-differentiable) $\tilde{y}, \tilde{\bar{y}}:\left[x_{0}, x_{0}+r\right] \rightarrow B\left(y_{0}, q\right)$ where $r=\min \left\{p, \frac{q}{M}, \frac{q}{M_{1}}, d\right\}$ and the successive iterations $\widetilde{y}_{0}(x)=\widetilde{y}_{0}, \widetilde{y}_{n+1}(x)=\widetilde{y}_{0}+\int_{x_{0}}^{x} \widetilde{f}\left(t, \widetilde{y}_{n}(t)\right) d t$ or $\widetilde{\bar{y}}_{0}(x)=\widetilde{\bar{y}}_{0}, \widetilde{\bar{y}}_{n+1}(x)=\widetilde{\bar{y}}_{0} \ominus(-1) \int_{x_{0}}^{x} \widetilde{f}\left(t, \widetilde{\bar{y}}_{n}(t)\right) d t$ converge to these two solutions, respectively.

\section{The basic idea of the technique}

The proposed technique comprises of the Adomian Decomposition Method and the Green's function. In order to solve second-order fuzzy boundary value problem (1) and (2) via the ADM, the original problem has to be divided into two sub-problems is divided. Firstly, the domain $\left[x_{0}, x_{2}\right]$ is divided into two sub-domains then the $\left[x_{0}, x_{1}\right] \cup\left[x_{1}, x_{2}\right]$ is decomposed,then set the $\widetilde{y}\left(x_{1}\right)=\gamma, x_{1} \in\left(x_{0}, x_{2}\right)$ where the $\gamma$ is an unknown fuzzy constant value. 
For $\left[x_{0}, x_{1}\right]$ : Consider the following second-order fuzzy boundary value problem

$$
\widetilde{y}^{\prime \prime}=F\left(x, \widetilde{y}(x), \widetilde{y}^{\prime}(x)\right), \widetilde{y}^{\prime}\left(x_{0}\right)=A, \widetilde{y}\left(x_{1}\right)=\gamma
$$

Integrating twice the problem (3) and applying $\vec{y}^{\prime}\left(x_{0}\right)=A$, allows for the Volterra integral equation to be obtained

$$
\left.\widetilde{y}(x)=\widetilde{y}\left(x_{0}\right)+A\left(x-x_{0}\right)+\int_{x_{0}}^{x}(x-\eta) f(\eta, \widetilde{y}(\eta)), \tilde{y}^{\prime}(\eta)\right) d \eta
$$

Using conditions from $\widetilde{y}\left(x_{1}\right)=\gamma, \widetilde{y}\left(x_{0}\right)$ is found as an

$$
\left.\widetilde{y}\left(x_{0}\right)=\gamma-A\left(x_{1}-x_{0}\right)+\int_{x_{0}}^{x_{1}}\left(x_{1}-\eta\right) f(\eta, \widetilde{y}(\eta)), \tilde{y}^{\prime}(\eta)\right) d \eta
$$

Substituting $\widetilde{y}\left(x_{0}\right)$ in Eq. (4), the Fredholm integral equation is obtained as this

$$
\left.\widetilde{y}(x)=\gamma+A\left(x-x_{1}\right)+\int_{x_{0}}^{x_{1}} G(x, \eta) f(\eta, \widetilde{y}(\eta)), \tilde{y}^{\prime}(\eta)\right) d \eta
$$

while, the Green's function $G(x, \eta)$ become this

$$
\begin{gathered}
G(x, \eta)= \begin{cases}\eta-x_{1}, & x_{0} \leq x \leq \eta, \\
x-x_{1}, & \eta \leq x \leq x_{1}\end{cases} \\
\left.\tilde{y}(x)=\sum_{j=0}^{\infty} \widetilde{y}_{j}(x), \quad f(x, \tilde{y}(x)), \tilde{y}(x)\right)=\sum_{j=0}^{\infty} \widetilde{A}_{j}
\end{gathered}
$$

is an infinite series where $\widetilde{A}_{j}$ has decomposed Adomian polynomials. Substituting (8) into (6),

$$
\sum_{j=0}^{\infty} \widetilde{y}_{j}(x)=\gamma+A\left(x-x_{1}\right)+\int_{x_{0}}^{x_{1}} G(x, \eta)\left[\sum_{j=0}^{\infty} \widetilde{A}_{j}\right] d \eta
$$

is obtained. Comparing both sides of (9),

$$
\left\{\begin{array}{l}
\widetilde{y}_{0}(x, \gamma)=\gamma+A\left(x-x_{1}\right), \\
\widetilde{y}_{j}(x, \gamma)=\int_{x_{0}}^{x_{1}} G(x, \eta) \widetilde{A}_{j-1} d \eta, \quad j=1,2, \ldots
\end{array}\right.
$$

is obtained and then the modified recursive scheme is defined as an

$$
\left\{\begin{array}{l}
\widetilde{y}_{0}(x, \gamma)=\gamma \\
\widetilde{y}_{1}(x, \gamma)=A\left(x-x_{1}\right)+\int_{x_{0}}^{x_{1}} G(x, \eta) \widetilde{A}_{0} d \eta \\
\widetilde{y}_{j}(x, \gamma)=\int_{x_{0}}^{x_{1}} G(x, \eta) \widetilde{A}_{j-1} d \eta, \quad j=2,3, \ldots
\end{array}\right.
$$

which allows a complete determination of the components in the $\tilde{y}_{j}(x)$. Followed by the $n$-terms series solution of the sub-problem (3) is given by

$$
\widetilde{\Phi}_{n}^{(1)}(x)=\sum_{j=0}^{n} \widetilde{y}_{j}(x, \gamma)
$$


From (7) Definition, it is possible to obtain the approximate solution of Eq. (1)-(2) in the following cases [8]. Case (a): If $\tilde{y}^{\prime}$ and $\widetilde{y}^{\prime \prime}$ are $(i)$-differentiable, Eq.(11) becomes

$$
\left\{\begin{array}{l}
\widetilde{y}_{0}(x, \gamma)=\gamma \\
\widetilde{y}_{1}(x, \gamma)=A\left(x-x_{1}\right)+\int_{x_{0}}^{x_{1}} G(x, \eta) \widetilde{A}_{0} d \eta \\
\widetilde{y}_{j}(x, \gamma)=\int_{x_{0}}^{x_{1}} G(x, \eta) \widetilde{A}_{j-1} d \eta, \quad \eta=2,3, \ldots
\end{array}\right.
$$

Case (b): If $\widetilde{y}$ and $\widetilde{y}^{\prime \prime}$ are (ii)-differentiable, Eq.(11) becomes

$$
\left\{\begin{array}{l}
\widetilde{y}_{0}(x, \gamma)=\gamma \\
\widetilde{y}_{1}(x, \gamma)=\ominus(-1)\left[A\left(x-x_{1}\right) \ominus(-1) \int_{x_{0}}^{x_{1}} G(x, \eta) \widetilde{A}_{0} d \eta\right], \\
\widetilde{y}_{j}(x, \gamma)=\ominus(-1)\left[\ominus(-1) \int_{x_{0}}^{x_{1}} G(x, \eta) \widetilde{A}_{j-1} d \eta\right], \quad \eta=2,3, \ldots
\end{array}\right.
$$

Case (c): If $\widetilde{y}^{\prime}$ is the $(i)$-differentiable and $\widetilde{y}^{\prime \prime}$ is the $(i i)$-differentiable, Eq.(11) becomes

$$
\left\{\begin{array}{l}
\widetilde{y}_{0}(x, \gamma)=\gamma \\
\widetilde{y}_{1}(x, \gamma)=\ominus(-1)\left[A\left(x-x_{1}\right)+\int_{x_{0}}^{x_{1}} G(x, \eta) \widetilde{A}_{0} d \eta\right] \\
\widetilde{y}_{j}(x, \gamma)=\ominus(-1)\left[\int_{x_{0}}^{x_{1}} G(x, \eta) \widetilde{A}_{j-1} d \eta\right], \quad \eta=2,3, \ldots
\end{array}\right.
$$

Case $(d)$ : If $\widetilde{y}^{\prime}$ is the (ii)-differentiable and $\widetilde{y}^{\prime \prime}$ is the $(i)$-differentiable, Eq.(11) becomes

$$
\left\{\begin{array}{l}
\tilde{y}_{0}(x, \gamma)=\gamma \\
\tilde{y}_{1}(x, \gamma)=A\left(x-x_{1}\right) \ominus(-1)\left[\int_{x_{0}}^{x_{1}} G(x, \eta) \widetilde{A}_{0} d \eta\right] \\
\widetilde{y}_{j}(x, \gamma)=\ominus(-1)\left[\int_{x_{0}}^{x_{1}} G(x, \eta) \widetilde{A}_{j-1} d \eta\right], \quad \eta=2,3, \ldots
\end{array}\right.
$$

For $\left[x_{1}, x_{2}\right]$ : Let us consider the following fuzzy boundary value problem

$$
\widetilde{y}^{\prime \prime}=F\left(x, \tilde{y}(x), \tilde{y}^{\prime}(x)\right), \widetilde{y}\left(x_{1}\right)=\gamma, \widetilde{y}^{\prime}(b)=B
$$

Integrating twice the problem (13) and applying an $\widetilde{y}\left(x_{1}\right)=\gamma$, the Volterra integral equation is obtained

$$
\left.\widetilde{y}(x)=\gamma+B\left(x-x_{0}\right)+\int_{x_{1}}^{x}(x-\eta) f(\eta, \widetilde{y}(\eta)), \tilde{y}^{\prime}(\eta)\right) d \eta
$$

Using the other boundary condition $\widetilde{y}^{\prime}\left(x_{2}\right)=B$, the following is yilded

$$
\left.\widetilde{y}^{\prime}\left(x_{1}\right)=B-\int_{x_{1}}^{x_{2}} f(\eta, \widetilde{y}(\eta)), \widetilde{y}^{\prime}(\eta)\right) d \eta
$$

Combining Eqs. (14) and (15), the Fredholm integral equation is obtained as

$$
\left.\widetilde{y}(x)=\gamma+B\left(x-x_{1}\right)+\int_{x_{1}}^{x_{2}} G(x, \eta) f(\eta, \widetilde{y}(\eta)), \tilde{y}^{\prime}(\eta)\right) d \eta
$$

where $G(x, \eta)$ is given by

$$
G(x, \eta)= \begin{cases}x_{1}-x, & x_{1} \leq x \leq \eta \\ x_{1}-\eta, & \eta \leq x \leq x_{2}\end{cases}
$$

By substituting (8) into (16),

$$
\sum_{j=0}^{\infty} \widetilde{y}_{j}(x)=\gamma+B\left(x-x_{1}\right)+\int_{x_{1}}^{x_{2}} G(x, \eta)\left[\sum_{j=0}^{\infty} \widetilde{A}_{j}\right] d \eta
$$


the above equation is obtained. From (18), the following recursion scheme occurs

$$
\left\{\begin{array}{l}
\widetilde{y}_{0}(x, \gamma)=\gamma+B\left(x-x_{1}\right), \\
\widetilde{y}_{j}(x, \gamma)=\int_{x_{1}}^{x_{2}} G(x, \eta) \widetilde{A}_{j-1} d \eta, \quad j=1,2, \ldots
\end{array}\right.
$$

and the modified recursive scheme as

$$
\left\{\begin{array}{l}
\widetilde{y}_{0}(x, \gamma)=\gamma \\
\widetilde{y}_{1}(x, \gamma)=B\left(x-x_{1}\right)+\int_{x_{1}}^{x_{2}} G(x, \eta) \widetilde{A}_{0} d \eta \\
\widetilde{y}_{j}(x, \gamma)=\int_{x_{1}}^{x_{2}} G(x, \eta) \widetilde{A}_{j-1} d \eta, \quad j=2,3, \ldots
\end{array}\right.
$$

is defined. Denote the $n$-terms approximant of the series solutions by using this

$$
\widetilde{\Phi}_{n}^{(2)}(x)=\sum_{j=0}^{n} \widetilde{y}_{j}(x, \gamma)
$$

From Definition (7), following cases exist [8].

Case (a): If $\tilde{y}^{\prime}$ and $\tilde{y}^{\prime \prime}$ are $(i)$-differentiable, Eq.(20) becomes

$$
\left\{\begin{array}{l}
\widetilde{y}_{0}(x, \gamma)=\gamma \\
\widetilde{y}_{1}(x, \gamma)=B\left(x-x_{1}\right)+\int_{x_{1}}^{x_{2}} G(x, \eta) \widetilde{A}_{0} d \eta \\
\widetilde{y}_{j}(x, \gamma)=\int_{x_{1}}^{x_{2}} G(x, \eta) \widetilde{A}_{j-1} d \eta, \quad j=2,3, \ldots
\end{array}\right.
$$

Case (b): If $\widetilde{y}^{\prime}$ and $\widetilde{y}^{\prime \prime}$ are (ii)-differentiable, Eq.(20) becomes

$$
\left\{\begin{array}{l}
\widetilde{y}_{0}(x, \gamma)=\gamma \\
\widetilde{y}_{1}(x, \gamma)=\ominus(-1)\left[B\left(x-x_{1}\right) \ominus(-1) \int_{x_{1}}^{x_{2}} G(x, \eta) \widetilde{A}_{0} d \eta\right] \\
\widetilde{y}_{j}(x, \gamma)=\ominus(-1)\left[\ominus(-1) \int_{x_{1}}^{x_{2}} G(x, \eta) \widetilde{A}_{j-1} d \eta\right], \quad j=2,3, \ldots
\end{array}\right.
$$

Case (c): If $\widetilde{y}$ is the $(i)$-differentiable and $\widetilde{y}^{\prime \prime}$ is the (ii)-differentiable, Eq.(20) becomes

$$
\left\{\begin{array}{l}
\widetilde{y}_{0}(x, \gamma)=\gamma \\
\widetilde{y}_{1}(x, \gamma)=\ominus(-1)\left[B\left(x-x_{1}\right)+\int_{x_{1}}^{x_{2}} G(x, \eta) \widetilde{A}_{0} d \eta\right] \\
\widetilde{y}_{j}(x, \gamma)=\ominus(-1)\left[\int_{x_{1}}^{x_{2}} G(x, \eta) \widetilde{A}_{j-1} d \eta\right], \quad j=2,3, \ldots
\end{array}\right.
$$

Case $(d)$ : If $\tilde{y}^{\prime}$ is the (ii)-differentiable and $\widetilde{y}^{\prime \prime}$ is the $(i)$-differentiable, Eq.(20) becomes

$$
\left\{\begin{array}{l}
\widetilde{y}_{0}(x, \gamma)=\gamma \\
\widetilde{y}_{1}(x, \gamma)=B\left(x-x_{1}\right) \ominus(-1)\left[\int_{x_{1}}^{x_{2}} G(x, \eta) \widetilde{A}_{0} d \eta\right] \\
\widetilde{y}_{j}(x, \gamma)=\ominus(-1)\left[\int_{x_{1}}^{x_{2}} G(x, \eta) \widetilde{A}_{j-1} d \eta\right], \quad j=2,3, \ldots
\end{array}\right.
$$

From Eqs. (12) and (21), the continuity condition to determine this $\gamma$ is used as follows:

$$
\left.\frac{d \widetilde{\Phi}_{n}^{(1)}(x, \gamma)}{d x}\right|_{x=x_{1}}-\left.\frac{d \widetilde{\Phi}_{n}^{(2)}(x, \gamma)}{d x}\right|_{x=x_{1}}=0, \quad n=1,2, \ldots
$$


From this, the approximate value of $\gamma$ is found. Then the approximate series solution of original second-order fuzzy boundary value problem can be found. (1) - (2) is defined as

$$
\widetilde{\Phi}_{n}(x)= \begin{cases}\widetilde{\Phi}_{n}^{(1)}\left(x, \gamma_{n}\right):=\sum_{j=0}^{\infty} \widetilde{y}_{j}\left(x, \gamma_{n}\right), & x_{0} \leq x \leq x_{1}, \\ \widetilde{\Phi}_{n}^{(2)}\left(x, \gamma_{n}\right):=\sum_{j=0}^{\infty} \widetilde{y}_{j}\left(x, \gamma_{n}\right), & x_{1} \leq x \leq x_{2}\end{cases}
$$

where the $\gamma_{n}, \quad n=1,2,3, \ldots$ approximates the values of $\gamma$ for each of case $(a)-(d)$.

\section{Convergence analysis}

In order to prove convergence using the proposed technique following assumptions are necessary [8].

For $\left[x_{0}, x_{1}\right]$ : Consider $\widetilde{f}(x)$ is bounded for all $x \in\left[x_{0}, x_{1}\right]$ and $\exists M_{1}, M_{2}>0$ where $\int_{x_{0}}^{x_{1}} \mid G\left(x, \eta \mid d \eta \leq M_{1}\right.$, $\int_{x_{0}}^{x_{1}} \mid G_{x}\left(x, \eta \mid d \eta \leq M_{2}\right.$. Supposes that the nonlinear operators $f\left(x, y, y^{\prime}\right)$ satisfies the Lipschitz conditions with

$$
D\left(f\left(x, y, y^{\prime}\right), f\left(x, z, z^{\prime}\right)\right) \leq L_{1} D(y, z)+L_{2} D\left(y^{\prime}, z^{\prime}\right), L_{1}>0, L_{2}>0
$$

If $\delta:=\left(L_{1} M_{1}+L_{2} M_{2}\right)<1$, then the Eq. (6) has a unique solution

Lemma 2.[8] If $\widetilde{u}, \widetilde{v}, \widetilde{w} \in F$ and $\lambda \in R$, then

(1) $D(\widetilde{u} \ominus \widetilde{v}, \widetilde{u} \ominus \widetilde{w})=D(\widetilde{v}, \widetilde{w})$

(2) $D(\ominus \lambda \widetilde{u}, \ominus \lambda \widetilde{v})=|\lambda| D(\widetilde{u}, \widetilde{v})$.

Theorem 2.[8] Let $0<\delta<1$, then Eqs. (1) - (2) have a unique solution where $\vec{y}^{\prime}$ (1)-differentiable and $\tilde{y}^{\prime \prime}$ is (2)differentiable, respectively.

Proof. Let $\widetilde{y}$ and $\widetilde{y}^{*}$ be two different solutions for (1) - (2), then

$D\left(\widetilde{y}(x), \widetilde{y}^{*}(x)\right)=D\left(A\left(x-x_{1}\right) \ominus(-1) \int_{x_{0}}^{x_{1}} G(x, \eta) f\left(\eta, \widetilde{y}(\eta), \widetilde{y}^{\prime}(\eta)\right) d \eta+\gamma\right.$,

$\left.A\left(x-x_{1}\right) \ominus(-1) \int_{x_{0}}^{x_{1}} G(x, \eta) f\left(\eta, \widetilde{y}^{*}(\eta), \widetilde{y}^{*}(\eta)\right) d \eta+\gamma\right)$

$=D\left(\ominus(-1) \int_{x_{0}}^{x_{1}} G(x, \eta) f(\eta, \widetilde{y}(\eta), \widetilde{y}(\eta)) d \eta, \ominus(-1) \int_{x_{0}}^{x_{1}} G(x, \eta) f\left(\eta, \widetilde{y}^{*}(\eta), \widetilde{y}^{*}(\eta)\right) d \eta\right)$

$=D\left(\int_{x_{0}}^{x_{1}} G(x, \eta) f(\eta, \widetilde{y}(\eta), \widetilde{y}(\eta)) d \eta, \int_{x_{0}}^{x_{1}} G(x, \eta) f\left(\eta, \widetilde{y}^{*}(\eta), \widetilde{y}^{*}(\eta)\right) d \eta\right)$

$\leq D\left(\int_{x_{0}}^{x_{1}} G(x, \eta) f(\eta, \widetilde{y}(\eta), \widetilde{y}(\eta)) d \eta\right)+D\left(\int_{x_{0}}^{x_{1}} G(x, \eta) f\left(\eta, \widetilde{y}^{*}(\eta), \widetilde{y}^{*}(\eta)\right) d \eta\right)$

$\leq\left(L_{1} M_{1}+L_{2} M_{2}\right) D\left(\widetilde{y}(x), \widetilde{y}^{*}(x)\right)=\delta D\left(\widetilde{y}(x), \widetilde{y}^{*}(x)\right)$.

From which $(1-\delta) D\left(\widetilde{y}(x), \widetilde{y}^{*}(x)\right) \leq 0$ is obtained. Since $0<\delta<1$, then $D\left(\widetilde{y}(x), \widetilde{y}^{*}(x)\right)=0$ implies $\widetilde{y}(x)=\widetilde{y}^{*}(x)$.

Theorem 3. Let $\widetilde{y}_{0}, \widetilde{y}_{1}, \widetilde{y}_{2}, \ldots$ be the solution components that were obtained by using the recursive schemes (10) and (11), and let $\widetilde{\Phi}_{n}=\sum_{j=0}^{n} \widetilde{y}_{j}$ be the $n$-terms series solution defined by (12). Then $\widetilde{\Phi}_{n}$ converges to the exact solution $\widetilde{y}$ whenever $\delta<1$.

Proof. Using (10), (11) and (12), we have

$\widetilde{\Phi}_{n}=\widetilde{y}_{0}+\sum_{j=1}^{n} \widetilde{y}_{j}$

$=\gamma+A\left(x-x_{1}\right)+\sum_{j=1}^{n}\left(\int_{x_{0}}^{x_{1}} G(x, \eta) \tilde{A}_{j-1} d \eta\right)$

$=\gamma+A\left(x-x_{1}\right)+\int_{x_{0}}^{x_{1}} G(x, \eta) \sum_{j=0}^{n-1} \widetilde{A}_{j} d \eta$. 
For all $n, m \in N$ with $n>m$, consider

$D\left(\widetilde{\Phi}_{n}, \widetilde{\Phi}_{m}\right) \leq \max _{x_{0} \leq x \leq x_{1}}\left|\int_{x_{0}}^{x_{1}} G(x, \eta)\left[\sum_{j=0}^{n-1} \widetilde{A}_{j}-\sum_{j=0}^{m-1} \widetilde{A}_{j}\right] d \eta\right|$.

Using the related $\sum_{j=0}^{n-1} \widetilde{A}_{j} \leq f\left(\eta, \widetilde{\Phi}_{n-1}, \widetilde{\Phi}_{n-1}^{\prime}\right)$ in $[28,29]$.

$D\left(\widetilde{\Phi}_{n}, \widetilde{\Phi}_{m}\right) \leq \max _{x_{0} \leq x \leq x_{1}}\left|\int_{x_{0}}^{x_{1}} G(x, \eta)\left[f\left(\eta, \widetilde{\Phi}_{n-1}, \widetilde{\Phi}_{n-1}^{\prime}\right)-f\left(\eta, \widetilde{\Phi}_{m-1}, \widetilde{\Phi}_{m-1}^{\prime}\right)\right] d \eta\right|$.

Allows for, the following relation to be obtained $D\left(\widetilde{\Phi}_{n+1}, \widetilde{\Phi}_{n}\right) \leq \delta D\left(\widetilde{\Phi}_{n}, \widetilde{\Phi}_{n-1}\right)$. Thus, it becomes

$D\left(\widetilde{\Phi}_{n+1}, \widetilde{\Phi}_{n}\right) \leq \delta D\left(\widetilde{\Phi}_{n}, \widetilde{\Phi}_{n-1}\right) \leq \delta^{2} D\left(\widetilde{\Phi}_{n-1}, \widetilde{\Phi}_{n-2}\right) \leq \ldots \leq \delta^{n} D\left(\widetilde{\Phi}_{1}, \widetilde{\Phi}_{0}\right)$.

For any $n, m \in N$, with $n>m$, consider

$D\left(\widetilde{\Phi}_{n}, \widetilde{\Phi}_{m}\right) \leq D\left(\widetilde{\Phi}_{n}, \widetilde{\Phi}_{n-1}\right)+D\left(\widetilde{\Phi}_{n-1}, \widetilde{\Phi}_{n-1}\right)+\ldots+D\left(\widetilde{\Phi}_{m+1}, \widetilde{\Phi}_{m}\right)$

$\leq\left[\delta^{n-1}+\delta^{n-2}+\ldots+\delta^{m}\right] D\left(\widetilde{\Phi}_{1}, \widetilde{\Phi}_{0}\right)=\delta^{m}\left(\frac{1-\delta^{n-m}}{1-\delta}\right) D\left(\widetilde{\Phi}_{1}, \widetilde{\Phi}_{0}\right)$

Since $\delta<1$, therefore $1-\delta^{n-m}<1$ and $D\left(\widetilde{\Phi}_{1}, \widetilde{\Phi}_{0}\right)<\infty$, follows that

$D\left(\widetilde{\Phi}_{n}, \widetilde{\Phi}_{m}\right)<\left(\frac{\delta^{m}}{1-\delta}\right) D\left(\widetilde{\Phi}_{1}, \widetilde{\Phi}_{0}\right)<\infty$, as $m \rightarrow \infty$.

This implies that there exists an $\widetilde{\Phi}$ such that $\lim _{n \rightarrow \infty} \widetilde{\Phi}_{n}=\widetilde{\Phi}$. Since, $\widetilde{y}=\sum_{j=0}^{\infty} \widetilde{y}_{j}=\lim _{n \rightarrow \infty} \widetilde{\Phi}_{n}$, has that is , $\widetilde{y}=\widetilde{\Phi}$

Remark. The proof of other cases is similar to the previous theorems. Also, similar steps for the recursive schemes (19) and (20) can be applied.

\section{Numerical results}

In this section, second-order fuzzy boundary value problem is solved under generalized differentiability using ADM with Green's function. All symbolic and numerical computations were created using MATLAB Software Package.

Example 1. Consider the following second-order fuzzy boundary value problem

$$
\left\{\begin{array}{l}
\widetilde{y}^{\prime \prime}(x)=-\left(2-4 x^{2}\right) \widetilde{y}, \quad x \in[0,1] \\
\widetilde{y}^{\prime}(0)=[0,0], \quad \widetilde{y}^{\prime}(1)=[-2 \exp (-1)(\alpha-1),-2 \exp (-1)(1-\alpha)]
\end{array}\right.
$$

where the exact solution is $\widetilde{y}(x)=\left[(\alpha-1) \exp \left(-x^{2}\right),(1-\alpha) \exp \left(-x^{2}\right)\right]$. To obtain approximate solution of above example, the ADM with Green's function is applied. Firstly, the domain $[0,1]$ is divided into domains $[0,0.5]$ and $[0.5,1]$. Suppose that $\widetilde{y}(0.5)=[\underline{\gamma}, \bar{\gamma}]$, where $\gamma$ is an unknown fuzzy constant.

Solve the following sub-problems

$$
\left\{\begin{array}{l}
\tilde{y}^{\prime \prime}(x)=-\left(2-4 x^{2}\right) \widetilde{y} \\
\tilde{y}^{\prime}(0)=[0,0], \widetilde{y}(0.5)=[\underline{\gamma}, \bar{\gamma}], \quad x \in[0,0.5]
\end{array}\right.
$$

and

$$
\left\{\begin{array}{l}
\tilde{y}^{\prime \prime}(x)=-\left(2-4 x^{2}\right) \tilde{y} \\
\widetilde{y}(0.5)=[\underline{\gamma}, \bar{\gamma}], \widetilde{y}(1)=[-2 \exp (-1)(\alpha-1),-2 \exp (-1)(1-\alpha)], \quad x \in[0.5,1]
\end{array}\right.
$$

Consider the following cases.

Case (a): If $\tilde{y}^{\prime}$ and $\tilde{y}^{\prime \prime}$ are $(i)$-differentiable,

For $[0,0.5]$ : According to the recursive scheme (11), the sub-problem (25) is transformed into

$\underline{y}_{0}(x)=\underline{\gamma}$

$\bar{y}_{0}(x)=\overline{\bar{\gamma}}$

$\underline{y}_{1}(x)=\frac{1}{48} \underline{\gamma}\left(16 x^{4}-48 x^{2}+11\right)$

$\bar{y}_{1}(x)=\frac{1}{48} \bar{\gamma}\left(16 x^{4}-48 x^{2}+11\right)$ 
Thus, the series solution can found using $\widetilde{\Phi}_{n}^{(1)}=\sum_{j=0}^{n} \widetilde{y}_{j}$.

For $[0.5,1]$ :According to the recursive scheme (20), the sub-problem (26) is transforms into

$\underline{y}_{0}(x)=\underline{\gamma}$

$\bar{y}_{0}(x)=\bar{\gamma}$

$\underline{y}_{1}(x)=(2 x-1)\left[\underline{\gamma}\left(0.1667 x^{3}+0.0833 x^{2}-0.4583 x+0.1042\right)+0.3679(1-\alpha)\right]$

$\bar{y}_{1}(x)=(2 x-1)\left[\bar{\gamma}\left(0.1667 x^{3}+0.0833 x^{2}-0.4583 x+0.1042\right)+0.3679(\alpha-1)\right]$

Thus, the series solution can found using $\widetilde{\Phi}_{n}^{(2)}=\sum_{j=0}^{n} \widetilde{y}_{j}$.

Case (b): If $\widetilde{y}^{\prime}$ and $\widetilde{y}^{\prime \prime}$ are (ii)-differentiable,

For $[0,0.5]$ : According to the recursive scheme (11), the sub-problem (25) is transformed into

$\underline{y}_{0}(x)=\bar{\gamma}$

$\bar{y}_{0}(x)=\underline{\gamma}$

$\underline{y}_{1}(x)=\frac{1}{48} \bar{\gamma}\left(16 x^{4}-48 x^{2}+11\right)$

$\bar{y}_{1}(x)=\frac{1}{48} \underline{\gamma}\left(16 x^{4}-48 x^{2}+11\right)$

Thus, the series solution can becomes $\widetilde{\Phi}_{n}^{(1)}=\sum_{j=0}^{n} \widetilde{y}_{j}$.

For $[0.5,1]$ :According to the recursive scheme (10), the sub-problem (26) is transformed using $\underline{y}_{0}(x)=\bar{\gamma}$

$\bar{y}_{0}(x)=\gamma$

$\underline{y}_{1}(x)=(2 x-1)\left[\bar{\gamma}\left(0.1667 x^{3}+0.0833 x^{2}-0.4583 x+0.1042\right)+0.3679(1-\alpha)\right]$

$\bar{y}_{1}(x)=(2 x-1)\left[\underline{\gamma}\left(0.1667 x^{3}+0.0833 x^{2}-0.4583 x+0.1042\right)+0.3679(\alpha-1)\right]$

Thus, the series solution is found as $\widetilde{\Phi}_{n}^{(2)}=\sum_{j=0}^{n} \widetilde{y}_{j}$.

Case (c): If $\vec{y}^{\prime}$ is the (i)-differentiable and $\widetilde{y}^{\prime \prime}$ is the (ii)-differentiable,

For $[0,0.5]$ : According to the recursive scheme (11), the sub-problem (25) is transformed using $\underline{y}_{0}(x)=\bar{\gamma}$

$\bar{y}_{0}(x)=\underline{\gamma}$

$\underline{y}_{1}(x)=-\frac{1}{48} \bar{\gamma}\left(16 x^{4}-48 x^{2}+11\right)$

$\bar{y}_{1}(x)=-\frac{1}{48} \underline{\gamma}\left(16 x^{4}-48 x^{2}+11\right)$

Thus, the series solution is found $\widetilde{\Phi}_{n}^{(1)}=\sum_{j=0}^{n} \widetilde{y}_{j}$.

For $[0.5,1]$ :According to the recursive scheme (10), the sub-problem (26) is transformed using $\underline{y}_{0}(x)=\bar{\gamma}$

$\bar{y}_{0}(x)=\underline{\gamma}$

$\underline{y}_{1}(x)=(1-2 x)\left[\bar{\gamma}\left(0.1667 x^{3}+0.0833 x^{2}-0.4583 x+0.1042\right)+0.3679(\alpha-1)\right]$

$\bar{y}_{1}(x)=(1-2 x)\left[\underline{\gamma}\left(0.1667 x^{3}+0.0833 x^{2}-0.4583 x+0.1042\right)+0.3679(1-\alpha)\right]$

Thus, the series solution is found as $\widetilde{\Phi}_{n}^{(2)}=\sum_{j=0}^{n} \widetilde{y}_{j}$.

Case $(d)$ : If $\tilde{y}^{\prime}$ is the (ii)-differentiable and $\widetilde{y}^{\prime \prime}$ is the $(i)$-differentiable,

For $[0,0.5]$ : According to the recursive scheme (11), the sub-problem (25) is transformed using $\underline{y}_{0}(x)=\underline{\gamma}$ 
$\bar{y}_{0}(x)=\bar{\gamma}$

$\underline{y}_{1}(x)=-\frac{1}{48} \underline{\gamma}\left(16 x^{4}-48 x^{2}+11\right)$

$\bar{y}_{1}(x)=-\frac{1}{48} \bar{\gamma}\left(16 x^{4}-48 x^{2}+11\right)$

:

Thus, the series solution is found as $\widetilde{\Phi}_{n}^{(1)}=\sum_{j=0}^{n} \widetilde{y}_{j}$.

For $[0.5,1]$ :According to the recursive scheme (10), the sub-problem (26) is transformed using

$\underline{y}_{0}(x)=\underline{\gamma}$

$\bar{y}_{0}(x)=\bar{\gamma}$

$\underline{y}_{1}(x)=(1-2 x)\left[\underline{\gamma}\left(0.1667 x^{3}+0.0833 x^{2}-0.4583 x+0.1042\right)+0.3679(1-\alpha)\right]$

$\bar{y}_{1}(x)=(1-2 x)\left[\overline{\bar{\gamma}}\left(0.1667 x^{3}+0.0833 x^{2}-0.4583 x+0.1042\right)+0.3679(\alpha-1)\right]$

:

Thus, the series solution can be found as $\widetilde{\Phi}_{n}^{(2)}=\sum_{j=0}^{n} \widetilde{y}_{j}$.

From Eq. (12), the numerical values for $[\underline{\gamma}, \bar{\gamma}]$ are listed in Table 1 when $\widetilde{y}^{\prime}$ and $\widetilde{y}^{\prime \prime}$ are $(i)$-differentiable. As seen from Table 1 , we observe that the numerical values of $[\underline{\gamma}, \bar{\gamma}]$ approaches the value $[(\alpha-1) 0.7788366,(1-\alpha) 0.7788366]$. The exact value of $[\underline{\gamma}, \bar{\gamma}]$ is $\widetilde{y}(0.5)=[(\alpha-1) \exp (-0.25),(1-\alpha) \exp (-0.25)]$. In Figure 1, the exact $\widetilde{y}(x)$ and the approximate

Table 1: Numerical values of $\left[\underline{\gamma}_{n}, \bar{\gamma}_{n}\right], n=2, \ldots, 6$ for $\alpha=0$.

\begin{tabular}{cccccc}
\hline$n$ & 2 & 3 & 4 & 5 & 6 \\
\hline$\underline{\gamma}_{n}$ & -0.7977789 & -0.7852824 & -0.7797234 & -0.7790055 & -0.7788366 \\
\hline $\bar{\gamma}_{n}$ & 0.7977789 & 0.7852824 & 0.7797234 & 0.7790055 & 0.7788366 \\
\hline
\end{tabular}

solutions $\widetilde{\Phi}_{n}, n=2, \ldots, 6$ for $\alpha=0$ when $\widetilde{y}^{\prime}$ and $\widetilde{y}^{\prime \prime}$ are $(i)$-differentiable is plotted.

The numerical values of $[\underline{\gamma}, \bar{\gamma}]$ are listed in Table 2 when $\widetilde{y}^{\prime}$ is $(i)$-differentiable and $\widetilde{y}^{\prime \prime}$ is $(i i)$-differentiable. In Figure

Fig. 1: Exact $\widetilde{y}(x)$ and the approximate $\widetilde{\Phi}_{n}, n=2, \ldots, 6$ solutions.

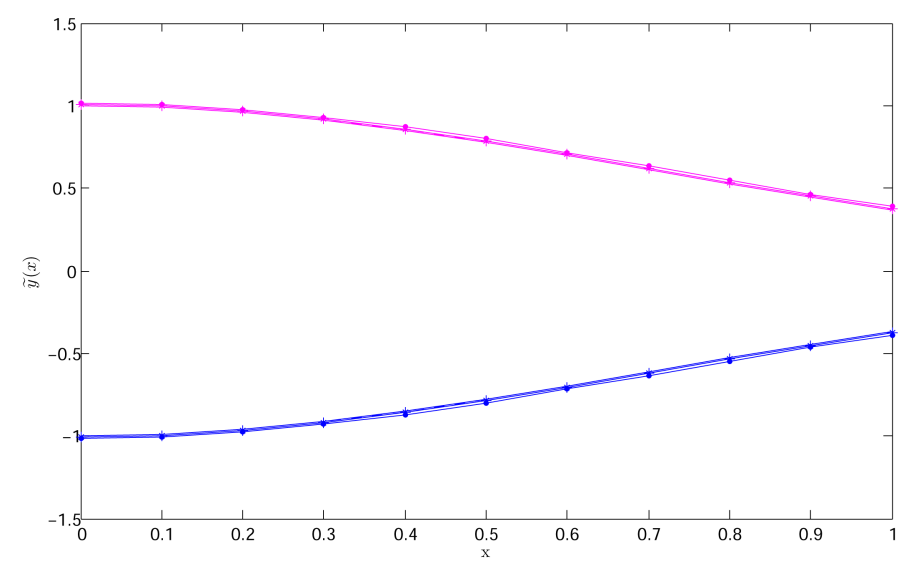

2, the approximate solutions $\widetilde{\Phi}_{n}, n=2, \ldots, 6$ for $\alpha=0$ when $\widetilde{y}^{\prime}$ is the $(i)$-differentiable and $\widetilde{y}^{\prime \prime}$ is the $(i i)$-differentiable is plotted. 
Table 2: Numerical values of $\left[\underline{\gamma}_{n}, \bar{\gamma}_{n}\right], n=2, \ldots, 6$ for $\alpha=0$

\begin{tabular}{cccccc}
\hline$n$ & 2 & 3 & 4 & 5 & 6 \\
\hline$\underline{\underline{\gamma}}_{n}$ & 0.7977789 & 0.7852824 & 0.7797234 & 0.7790055 & 0.7788366 \\
\hline $\bar{\gamma}_{n}$ & -0.7977789 & -0.7852824 & -0.7797234 & -0.7790055 & -0.7788366 \\
\hline
\end{tabular}

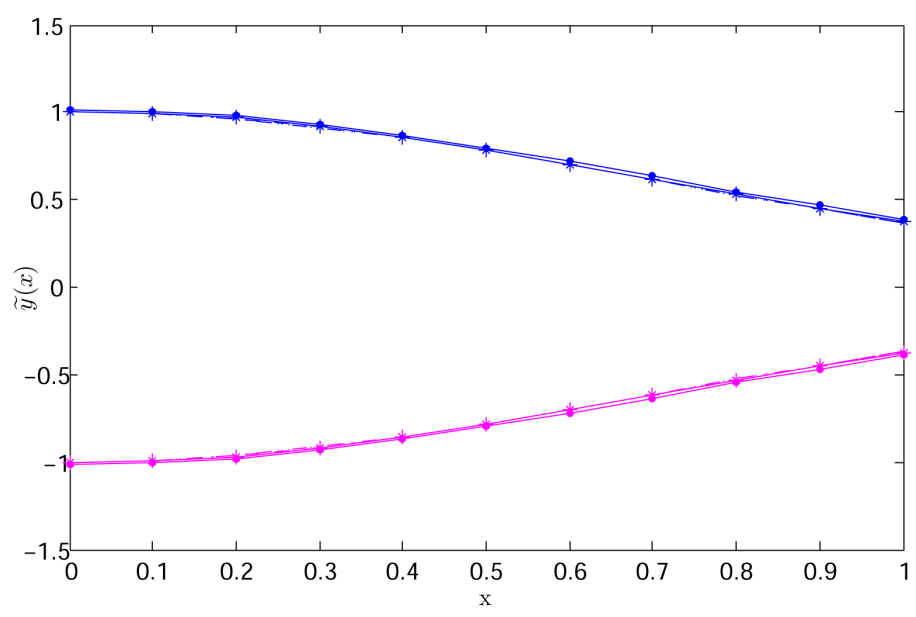

Fig. 2: Approximate $\widetilde{\Phi}_{n}, n=2, \ldots, 6$ solutions.

Example 2. Consider the following second-order fuzzy boundary value problem

$$
\left\{\begin{array}{l}
\widetilde{y}^{\prime \prime}(x)=2 \widetilde{y}^{3}, \quad x \varepsilon[0,1], \\
\left.\widetilde{y}^{\prime}(0)=[1-\alpha, \alpha-1], \quad \widetilde{y}^{\prime}(1)=\left[\frac{1}{4}(1-\alpha), \frac{1}{4}\right)(\alpha-1)\right]
\end{array}\right.
$$

where the exact solution is $\widetilde{y}(x)=\left[(\alpha-1) \frac{1}{1+x},(1-\alpha) \frac{1}{1+x}\right]$. The domain $[0,1]$ is divided into domains $[0,0.5]$ and $[0.5,1]$ and use $\widetilde{y}(0.5)=[\underline{\gamma}, \bar{\gamma}]$.

Solve the following problem

$$
\left\{\begin{array}{l}
\widetilde{y}^{\prime \prime}(x)=2 \widetilde{y}^{3}, \\
\widetilde{y}^{\prime}(0)=[1-\alpha, \alpha-1], \widetilde{y}(0.5)=[\underline{\gamma}, \bar{\gamma}], \quad x \in[0,0.5]
\end{array}\right.
$$

and

$$
\left\{\begin{array}{l}
\tilde{y}^{\prime \prime}(x)=2 \widetilde{y}^{3} \\
\left.\widetilde{y}(0.5)=[\underline{\gamma}, \bar{\gamma}], \widetilde{y}^{\prime}(1)=\left[\frac{1}{4}(1-\alpha), \frac{1}{4}\right)(\alpha-1)\right], \quad x \in[0.5,1]
\end{array}\right.
$$

Consider the following cases.

Case (a): If $\widetilde{y}^{\prime}$ and $\widetilde{y}^{\prime \prime}$ are $(i)$-differentiable,

For $[0,0.5]$ : According to the recursive scheme (11), the sub-problem (28) is transformed using

$\underline{y}_{0}(x)=\underline{\gamma}$
$\bar{y}_{0}(x)=\overline{\bar{\gamma}}$

$\underline{y}_{1}(x)=\underline{\gamma}^{3}\left[(2 x-1)-\frac{(2 x-1)^{2}}{4}\right]+(1-\alpha)\left(x-\frac{1}{2}\right)$

$\bar{y}_{1}(x)=\bar{\gamma}^{3}\left[(2 x-1)-\frac{(2 x-1)^{2}}{4}\right]+(\alpha-1)\left(x-\frac{1}{2}\right)$ 
Thus, the series solution can found using $\widetilde{\Phi}_{n}^{(1)}=\sum_{j=0}^{n} \widetilde{y}_{j}$.

For $[0.5,1]$ :According to the recursive scheme (10), the sub-problem (29) is transformed using $\underline{y}_{0}(x)=\underline{\gamma}$

$\bar{y}_{0}(x)=\bar{\gamma}$

$\underline{y}_{1}(x)=\underline{\gamma}^{3}\left[(2 x-1)(x-1)-\frac{(2 x-1)^{2}}{4}\right]+(1-\alpha)\left(\frac{x}{4}-\frac{1}{8}\right)$

$\bar{y}_{1}(x)=\bar{\gamma}^{3}\left[(2 x-1)(x-1)-\frac{(2 x-1)^{2}}{4}\right]+(\alpha-1)\left(\frac{x}{4}-\frac{1}{8}\right)$

Thus, the series solution is found using $\widetilde{\Phi}_{n}^{(2)}=\sum_{j=0}^{n} \widetilde{y}_{j}$.

Case (b): If $\widetilde{y}$ and $\widetilde{y}^{\prime \prime}$ are (ii)-differentiable,

For $[0,0.5]$ : According to the recursive scheme (11), the sub-problem (28) is transformed using $\underline{y}_{0}(x)=\bar{\gamma}$

$\bar{y}_{0}(x)=\underline{\gamma}$

$\underline{y}_{1}(x)=\bar{\gamma}^{3}\left[\frac{(2 x-1)^{2}}{4}-x(2 x-1)\right]+(\alpha-1)\left(x-\frac{1}{2}\right)$

$\bar{y}_{1}(x)=\underline{\gamma}^{3}\left[\frac{(2 x-1)^{2}}{4}-x(2 x-1)\right]+(1-\alpha)\left(x-\frac{1}{2}\right)$

:

Thus, the series solution can be found using $\widetilde{\Phi}_{n}^{(1)}=\sum_{j=0}^{n} \widetilde{y}_{j}$.

For $[0.5,1]$ :According to the recursive scheme (10), the sub-problem (29) is transformed using $\underline{y}_{0}(x)=\bar{\gamma}$

$\bar{y}_{0}(x)=\underline{\gamma}$

$\underline{y}_{1}(x)=\bar{\gamma}^{3}\left[\frac{(2 x-1)^{2}}{4}+(x-1)(2 x-1)\right]+(\alpha-1)\left(\frac{x}{4}-\frac{1}{8}\right)$

$\bar{y}_{1}(x)=\underline{\gamma}^{3}\left[\frac{(2 x-1)^{2}}{4}+(x-1)(2 x-1)\right]+(1-\alpha)\left(\frac{x}{4}-\frac{1}{8}\right)$

:

Thus, the series solution is found using $\widetilde{\Phi}_{n}^{(2)}=\sum_{j=0}^{n} \widetilde{y}_{j}$.

Case (c): If $\vec{y}^{\prime}$ is the $(i)$-differentiable and $\widetilde{y}^{\prime \prime}$ is the (ii)-differentiable,

For $[0,0.5]$ : According to the recursive scheme (11), the sub-problem (28) is transformed using $\underline{y}_{0}(x)=\bar{\gamma}$

$\bar{y}_{0}(x)=\underline{\gamma}$

$\underline{y}_{1}(x)=\bar{\gamma}^{3}\left[x(2 x-1)-\frac{(2 x-1)^{2}}{4}\right]+(\alpha-1)\left(x-\frac{1}{2}\right)$

$\bar{y}_{1}(x)=\underline{\gamma}^{3}\left[x(2 x-1)-\frac{(2 x-1)^{2}}{4}\right]+(1-\alpha)\left(x-\frac{1}{2}\right)$

Thus, the series solution is found using $\widetilde{\Phi}_{n}^{(1)}=\sum_{j=0}^{n} \widetilde{y}_{j}$.

For $[0.5,1]$ :According to the recursive scheme (10), the sub-problem (29) is transformed using $\underline{y}_{0}(x)=\bar{\gamma}$

$\bar{y}_{0}(x)=\underline{\gamma}$

$\underline{y}_{1}(x)=\bar{\gamma}^{3}\left[(x-1)(2 x-1)-\frac{(2 x-1)^{2}}{4}\right]+(\alpha-1)\left(\frac{x}{4}-\frac{1}{8}\right)$

$\bar{y}_{1}(x)=\underline{\gamma}^{3}\left[(x-1)(2 x-1)-\frac{(2 x-1)^{2}}{4}\right]+(1-\alpha)\left(\frac{x}{4}-\frac{1}{8}\right)$

$\vdots$

Thus, the series solution is found using $\widetilde{\Phi}_{n}^{(2)}=\sum_{j=0}^{n} \widetilde{y}_{j}$.

Case $(d)$ : If $\widetilde{y}^{\prime}$ is the (ii)-differentiable and $\widetilde{y}^{\prime \prime}$ is the $(i)$-differentiable,

For $[0,0.5]$ : According to the recursive scheme (11), the sub-problem (28) is transformed using 


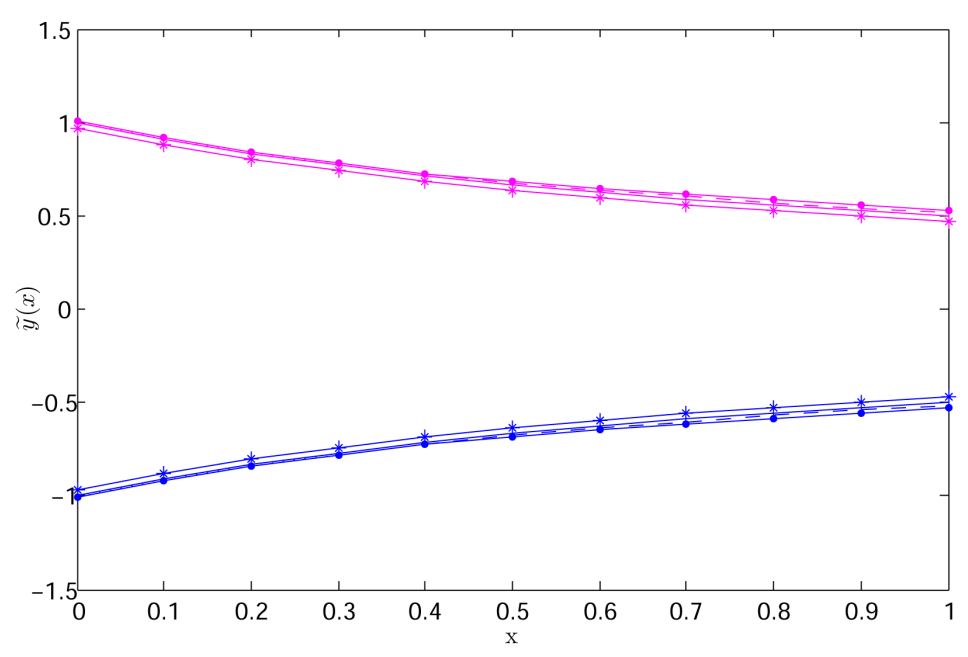

Fig. 3: Exact $\widetilde{y}(x)$ and the approximate $\widetilde{\Phi}_{n}, n=2, \ldots, 5$ solutions.

$\underline{y}_{0}(x)=\underline{\gamma}$

$\bar{y}_{0}(x)=\bar{\gamma}$

$\underline{y}_{1}(x)=\underline{\gamma}^{3}\left[\frac{(2 x-1)^{2}}{4}-x(2 x-1)\right]+(\alpha-1)\left(x-\frac{1}{2}\right)$

$\bar{y}_{1}(x)=\bar{\gamma}^{3}\left[\frac{(2 x-1)^{2}}{4}-x(2 x-1)\right]+(1-\alpha)\left(x-\frac{1}{2}\right)$

Thus, the series solution is found using $\widetilde{\Phi}_{n}^{(1)}=\sum_{j=0}^{n} \widetilde{y}_{j}$.

For $[0.5,1]$ :According to the recursive scheme (10), the sub-problem (29) is transformed using

$\underline{y}_{0}(x)=\underline{\gamma}$

$\bar{y}_{0}(x)=\bar{\gamma}$

$\underline{y}_{1}(x)=\underline{\gamma}^{3}\left[\frac{(2 x-1)^{2}}{4}-(x-1)(2 x-1)\right]+(\alpha-1)\left(\frac{x}{4}-\frac{1}{8}\right)$

$\bar{y}_{1}(x)=\bar{\gamma}^{3}\left[\frac{(2 x-1)^{2}}{4}-(x-1)(2 x-1)\right]+(1-\alpha)\left(\frac{x}{4}-\frac{1}{8}\right)$

Thus, the series solution is $\widetilde{\Phi}_{n}^{(2)}=\sum_{j=0}^{n} \widetilde{y}_{j}$.

As seen from Table 3, the numerical values of $[\underline{\gamma}, \bar{\gamma}]$ approaches the value $[(\alpha-1) 0.6673067,(1-\alpha) 0.0 .6673067]$ when $\tilde{y}^{\prime}$ and $\tilde{y}^{\prime \prime}$ are $(i)$-differentiable. Note that the exact value of $[\underline{\gamma}, \bar{\gamma}]$ is $\widetilde{y}(0.5)=\left[(\alpha-1) \frac{2}{3},(1-\alpha) \frac{2}{3}\right]$. In Figure 3 , the exact

Table 3: Numerical values of $\left[\underline{\gamma}_{n}, \bar{\gamma}_{n}\right], n=2, \ldots, 5$ for $\alpha=0$.

\begin{tabular}{ccccc}
\hline$n$ & 2 & 3 & 4 & 5 \\
\hline$\underline{\gamma}_{n}$ & -0.6825986 & -0.6382310 & -0.6789988 & -0.6673067 \\
\hline $\bar{\gamma}_{n}$ & 0.6825986 & 0.6382310 & 0.6789988 & 0.6673067 \\
\hline
\end{tabular}

$\widetilde{y}(x)$ and the approximate solutions $\widetilde{\Phi}_{n}, n=2, \ldots, 5$ for $\alpha=0$ when $\widetilde{y}^{\prime}$ and $\widetilde{y}^{\prime \prime}$ are $(i)$-differentiable is plotted.

The numerical values of $[\underline{\gamma}, \bar{\gamma}]$ are listed in Table $4 \alpha=0$ when $\tilde{y}^{\prime}$ is the $(i)$-differentiable and $\tilde{y}^{\prime \prime}$ is the $(i i)$-differentiable. In Figure 4, the approximate solutions $\widetilde{\Phi}_{n}, n=2, \ldots, 5$ for $\alpha=0$ when $\widetilde{y}^{\prime}$ is the $(i)$-differentiable and $\widetilde{y}^{\prime \prime}$ is the $(i i)$ differentiable is plotted. 
Table 4: Approximate value of $\left[\underline{\gamma}_{n}, \bar{\gamma}_{n}\right], n=2, \ldots, 5$ for $\alpha=0$

\begin{tabular}{ccccc}
\hline$n$ & 2 & 3 & 4 & 5 \\
\hline$\underline{\gamma}_{n}$ & 0.6825986 & 0.6382310 & 0.6789988 & 0.6673067 \\
\hline $\bar{\gamma}_{n}$ & -0.6825986 & -0.6382310 & -0.6789988 & -0.6673067 \\
\hline
\end{tabular}

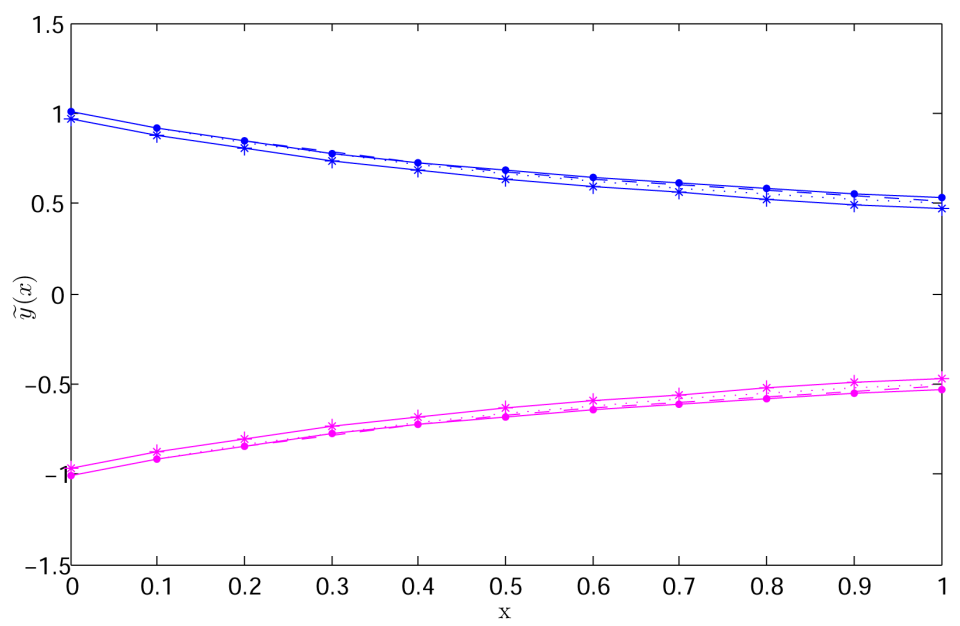

Fig. 4: Approximate $\widetilde{\Phi}_{n}, n=2, \ldots, 5$ solutions.

\section{Conclusion}

The ADM with Green's function for the solution of second-order fuzzy boundary value problem under generalized differentiability is presented. The proposed technique is interested in dividing the domain and building Green's function before installing the modified recursive scheme. The efficiency of the technique is illustrated by two numerical examples.

\section{Competing interests}

The authors declare that they have no competing interests.

\section{Authors' contributions}

All authors have contributed to all parts of the article. All authors read and approved the final manuscript.

\section{References}

[1] Akın Ö, Oruç Ö. A prey-predator model with fuzzy initial values. Hacet J Math Stat 2012; 41: 387-395.

[2] Allahviranloo T, Abbasbandy S, Sedaghgatfar O, Darabi P. A new method for solving fuzzy integro-differential equation under generalized differentiability. Neural Comput Appl 2012; 1: 191-196.

[3] Allahviranloo T, Amirteimoori A, Khezerloo M, Khezeloo S. A new method for solving fuzzy Volterra integro-differential equations. Aust J Basic Appl Sci 2011; 5: 154-164.

[4] Allahviranloo T, Khezerloo M, Ghanbari M, Khezerloo S. The homotopy perturbation method for fuzzy Volterra inetgral equations. Intern J Comp Cogn 2010; 8: 31-37. 
[5] Babolian E, Sadeghi GH, Abbasbandy S. Numerical solution of linear Fredholm fuzzy integral equations of the second kind by Adomian method. Appl Math Comput 2005; 161: 733-744.

[6] Bede B. A note on "Two-point boundary value problems associated with nonlinear fuzzy diffential equations". Fuzzy Set Syst 2006; 157: 986-989.

[7] Bede B, Gal SG. Generalizations of the differentiability of fuzzy-number-valued functions with applications to fuzzy differential equations. Fuzzy Set Syst 2005; 151: 581-599.

[8] Behzadi SS, Allahviranloo T, Abbasbandy S. Solving fuzzy second-order nonlinear Volterra-Fredholm integro-differential equqtions by using Picard method. Neural Comput Appl 2012; 1: 337-346.

[9] Can E, Bayrak MA. A New Method For Solution Of Fuzzy Reaction Equation. MATCH-Commun Math Co 2015; 74: 649-661.

[10] Chalco-Cano Y, Romacutean-Flores H. On new solutions of fuzzy differential equations. Chaos Soliton Fract 2008; 38 : 112-119.

[11] Chang SSL, Zadeh LA. On fuzzy mapping and control. IEEE T Syst Man Cyb 1972; 2: 30-34.

[12] Chen M, Fu Y, Xue X, Wu C. Two-point boundary value problems of undamped uncertain dynamical systems. Fuzzy Set Syst 2008; 159: 2077-2089.

[13] Chen M, Fu Y, Xue X, Wu C. On fuzzy boundary value problems. Inform Sciences 2008; 178: 1877-1892.

[14] Fatullayev AG, Koroglu C. Numerical solving a boundary value problem for fuzzy differential equations. CMES-Comput Model Eng 2012; 8: 39-52.

[15] Friedman M, Ma M, Kandel A. Numerical methods for calculating the fuzzy integral. Fuzzy Set Syst 1996; 83: 57-62.

[16] Gasilov NA, Hashimoglu IF, Amrahov SE, Fatullayev AG. A new approach to non-homogeneous fuzzy initial value problem. CMES-Comp Model Eng 2012; 85: 367-378.

[17] Goetschel R, Voxman W. Elementary calculus . Fuzzy Set Syst 1986; 18: 31-43.

[18] Jahantigh M, Allahviranloo T, Otadi M. Numerical solution of fuzzy integral equation. Appl Math Sci 2008; 2: 33-46.

[19] Kaleva O. Fuzzy Differential Equations. Fuzzy Set Syst 1987; 24: 301-317.

[20] Khastan A, Ivaz K. Numerical solution of fuzzy differential equations by Nyström method. Chaos Soliton Fract 2009; 41: 859-868.

[21] Khastan A, Nieto JJ. A boundary value problem for second-order fuzzy differential equations. Nonlinear Anal 2010; 72 : 35833593.

[22] Khastan A, Bahrami F, Ivaz K. New results on multiple solutions for Nth-order fuzzy differential equations under generalized differentiability. Bound Value Probl 13p,ARTICLE ID 395714, 2009.

[23] Khastan A, Nieto JJ, Lopes RR. Variation of constant formula for first order fuzzy differential equations. Fuzzy Set Syst 2011; 177: 20-33.

[24] Molabahrami A, Shidfar A, Ghyasi A. A An analytical method for solving linear Fredholm fuzzy integral equations of the second kind. Comput Math Appl 2011; 61: 2754-2761.

[25] Park JY, Lee SY, Jeong J. On the existence and uniqueness of solutions of fuzzy Volterra-Fredholm integral equations. Fuzzy Set Syst 2000; 115: 425-431.

[26] Puri M, Ralescu D. Differential and fuzzy functions. J Math Anal Appl 1983; 91: 552-558.

[27] Puri M, Ralescu D. Fuzzy Random variables. J Math Anal Appl 1986; 114: 409-422.

[28] Rach RC. A new definition of the Adomian polynomials. Kybernetes 2008; 37: 910-955.

[29] Singh R, Wazwaz A. An efficient approach for solving second-order nonlinear differential equation with Neumann boundary conditions. J Math Chem 2015; 53: 767-790.

[30] Zadeh LA. The concept of linguistic variable and its application to approximate reasoning. Inform Sciences 1975; 8: 199-249. 